\title{
The Use of an Interleukin-6 Inhibitor in Vasoplegic Shock from Severe Systemic Inflammatory Response Syndrome: A Case Report
}

\author{
Wiaam Y Elkhatib ${ }^{1} \odot$, Hollie Saunders ${ }^{2} \odot$, Scott A Helgeson ${ }^{3} \odot$, John E Moss ${ }^{4} \odot$
}

\begin{abstract}
A 66-year-old Caucasian male with a history of chronic myelomonocytic leukemia (CMML) developed fluid-unresponsive hypotension requiring initiation of four different maximum dosed vasopressors, steroids, and broad-spectrum antibiotics 4 hours following four-vessel coronary artery bypass grafting involving a 150-minute cardiac bypass. Placement of a Swanz-Ganz catheter showed a cardiac output of $7 \mathrm{~L} / \mathrm{min}$ ute with systemic vascular resistance of $571 \mathrm{dynes} / \mathrm{sec} / \mathrm{cm}^{-5}$. Over 24 hours, three doses of tocilizumab (interleukin- 6 inhibitor) every 8 hours were initiated, plus $250 \mathrm{mg}$ methylprednisolone per 6 hours increment, and then daily thereafter. After the initial dose of tocilizumab, it was possible to wean vasoconstrictors. We have shown for the first time that therapy with tocilizumab is effective in reversing the hemodynamic instability associated with the significant systemic inflammatory response from the "double hit" of CMML and coronary artery bypass grafting with cardiopulmonary bypass as has previously been shown in cytokine release syndrome.

Keywords: Cardiopulmonary bypass, Chronic myelomonocytic leukemia, Coronary artery bypass grafting, Cytokine release syndrome, Systemic inflammatory response syndrome, Tocilizumab.

Indian Journal of Critical Care Medicine (2021): 10.5005/jp-journals-10071-23943
\end{abstract}

\section{INTRODUCTION}

Chronic myelomonocytic leukemia (CMML) is a rare type of cellular dysplasia with around 1,100 new diagnoses annually in the United States, mostly in males aged above 60 years. ${ }^{1}$ Both abnormal increases in white blood cells and immature stem cell precursors are involved, clinically characterized by greater than 3 months of monocytosis ( $>1 \times 10^{9} / \mathrm{L}$ and greater than $10 \%$, normal being $0.30-0.90 \times 10^{9} / \mathrm{L}$ and between 2 and $8 \%$ ) along with biopsy findings of dysplastic bone marrow. ${ }^{1}$ The somatic mutation of TET2 is identified in roughly $60 \%$ of cases, which has been linked to increased inflammatory cytokine and chemokine expression. ${ }^{2}$ In these cases, patients will be in a constant proinflammatory state. Cardiovascular surgery is also known to induce an inflammatory state independently, through the release of tumor necrosis factor alpha (TNF-a) and specific interleukins (IL) including IL-6. $3,5,6$ Additional insults, such as cardiopulmonary bypass (CPB), can lead to a systemic and uncontrolled state of inflammation similar to cytokine release syndrome (CRS) in patients having pre-existing conditions such as severe viral infections or certain oncologic immunotherapies. ${ }^{3,4} \mathrm{IL}-6$ has been implicated to drive many of the CRS symptoms and, as such, tocilizumab is often administered for its properties of binding to and inhibiting IL- 6 receptor (soluble and membranous) signaling. Tocilizumab became standard for severe CRS management after demonstrating significant response in $69 \%$ of patients having severe cases. ${ }^{4}$

We present a case of tocilizumab use following cardiac surgery in a patient with existing CMML mimicking CRS.

\section{Case Description}

A 66-year-old male with a medical history of gastroesophageal reflux, CMML (low-risk dysplastic type, TET2/ZRSR2 pathogenic mutations), and no prior known cardiovascular history besides

\footnotetext{
1,2,4 Department of Internal Medicine, Mayo Clinic, Jacksonville, Florida, United States

${ }^{3}$ Department of Pulmonary and Critical Care Medicine, Mayo Clinic, Jacksonville, Florida, United States
}

Corresponding Author: Wiaam Y Elkhatib, Department of Internal Medicine, Mayo Clinic, Jacksonville, Florida, United States, e-mail: elkhatib.wiaam@mayo.edu

How to cite this article: Elkhatib WY, Saunders H, Helgeson SA, Moss JE. The Use of an Interleukin-6 Inhibitor in Vasoplegic Shock from Severe Systemic Inflammatory Response Syndrome: A Case Report. Indian J Crit Care Med 2021;25(8):939-941.

Source of support: Nil

Conflict of interest: None

hypertension was electively transferred from an outside facility on behalf of multivessel coronary artery disease continued management. Four days earlier, he developed chest pain of 12 hours due to non-ST elevation myocardial infarction. At the time, cardiac angiography indicated diffuse coronary artery disease including the right coronary and left main artery. Transthoracic echocardiography reported an ejection fraction of $49 \%$ with a small left ventricular size, but no regional wall motion abnormalities. Due to his multivessel disease, he was deemed not a candidate for percutaneous coronary intervention and thus offered coronary artery bypass graft (CABG) surgery. On admission to our facility, intravenous unfractionated heparin was initiated and his leukocyte count was $5.1 \times 10^{9} / \mathrm{L}$ (normal 3.5-10.5 $\times 10^{9} / \mathrm{L}$ ) with mild thrombocytopenia, which were both stable from previous laboratory data. Once the patient was medically optimized two days following admission, a four-vessel CABG was performed with a 150-minute CPB and no intraoperative complications. 
Immediately after surgery, he was able to be weaned from all vasopressors and was extubated within 2 hours of arriving to the intensive care unit. Two hours following completion of surgery he experienced rapidly progressive hypotension with MAP's via arterial line as low as $51 \mathrm{~mm} \mathrm{Hg}$. His hypotension was fluid resistant after being given $450 \mathrm{cc}$ cell saver with $2.4 \mathrm{~L}$ crystalloid intraoperatively, and $500 \mathrm{cc}$ albumin plus $500 \mathrm{cc}$ LR. The patient thus required the sequential initiation of four vasopressors (epinephrine, vasopressin, phenylephrine, norepinephrine). During this time he began to have worsening respiratory distress and was reintubated along with Swanz-Ganz catheter placement showing $\mathrm{CO}$ of $7 \mathrm{~L} /$ minute with systemic vascular resistance of 571 dynes $/ \mathrm{sec} / \mathrm{cm}^{-5}$ and arterial blood gas $\mathrm{pH}$ of 7.2, $\mathrm{pCO}_{2}$ of $45 \mathrm{~mm} \mathrm{Hg}$, and $\mathrm{PaO}_{2}$ of $110 \mathrm{~mm} \mathrm{Hg}$. Laboratory data revealed anemia (hemoglobin $7.6 \mathrm{gm} / \mathrm{dL}$ ), lactic acidosis (lactate $15 \mathrm{mmol} / \mathrm{L}$ ), and leukocytosis (white blood cell count $59.7 \times 10^{9} / \mathrm{L}$ ). Thiamine, desmopressin, methylene blue (two doses), acetylcysteine, phytonadione, vitamin B12, multiple doses of sodium bicarbonate and blood product transfusions (platelets, packed red blood cells, fresh frozen plasma, cryoprecipitate), stress-dose steroids with hydrocortisone $50 \mathrm{mg}$ every 6 hours, and empiric broad-spectrum antibiotics were administered with minimal to no improvement in hemodynamics. Transesophageal echocardiogram did not show any new cardiac dysfunction, and computed tomography imaging showed no acute concerns. Continuous renal replacement therapy (CRRT) and bicarbonate drip were required for refractory acidosis.

The patient was suspected to have developed severe vasoplegic shock mimicking CRS following extracorporeal circulation for his CABG. Supportive care was continued along with three doses of tocilizumab $(8 \mathrm{mg} / \mathrm{kg}$ ) every 8 hours. The stressdose steroids were switched to $250 \mathrm{mg}$ of methylprednisolone in 6-hour increments over a 24-hour period tapered down to daily thereafter. No leukapheresis was indicated given the lack of peripheral blasts despite predominant neutrophilia. After the initial tocilizumab dose on POD 1, vasopressors could begin to be weaned and by POD 4 from initiation of tocilizumab, the patient was no longer vasopressor dependent. With the continuation of tocilizumab, hemodynamics were stabilized and the patient was able to be extubated 8 days postsurgery (Fig. 1). Patient remained in the ICU 28 days postsurgery on CRRT until expiring due to other complications.

\section{Discussion}

CMML is linked to inflammatory marker elevations correlating with TET2 mutation (Fig. 2). ${ }^{2}$ It has also been demonstrated that surgeries requiring $\mathrm{CPB}$ or certain immunotherapies trigger proinflammatory processes leading to increased chemokine and cytokine release. In hosts having active pre-existing states of inflammation, such cascades may induce SIRS mimicking a CRS-type of reaction. Mild symptoms include fatigue, headache, and myalgia. Severe cases present with pyrexia, disseminated intravascular coagulation, hypotension with vasopressorrequiring vasoplegic shock, neurotoxicity, acute hypoxic respiratory failure, and multiorgan failure ${ }^{4}$ which further promotes leukocytosis in CMML. Core cytokines consistently described to be elevated in CRS patients are interferon (IFN)-Y, IL-10, and IL- 6 which particularly drives vascular leak and triggers the compliment cascade.

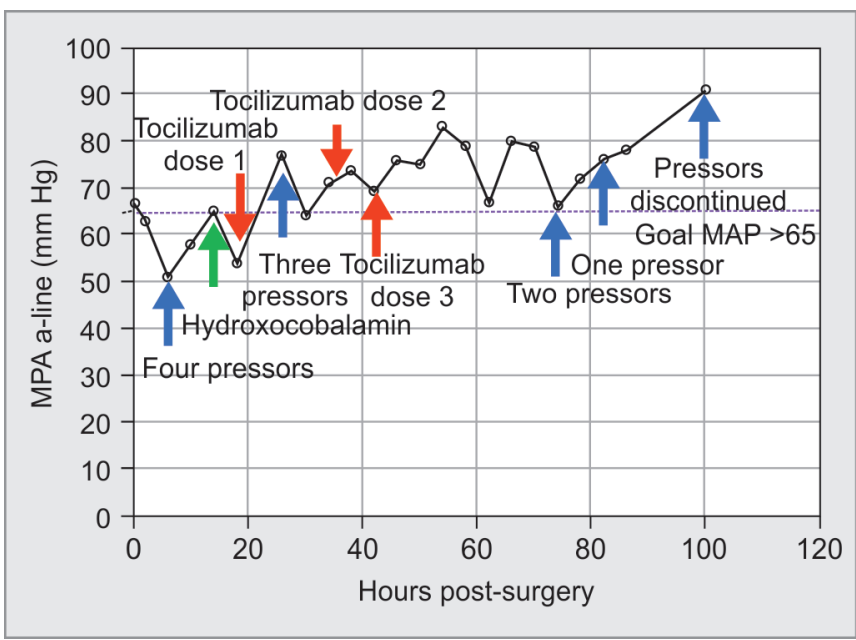

Fig. 1: Hemodynamic profile of patient after CABG. The plot in black shows progression of blood pressures approximately every 4 hours over the course of four days until pressors completely discontinued. $\mathrm{Y}$-axis shows blood pressure changes in $\mathrm{mm} \mathrm{Hg}$ and $\mathrm{X}$-axis shows hours postsurgery. Blue arrows represent times at which pressors were initiated and tracks the number of pressors required to maintain goal MAP > 65 (dashed horizontal purple line). Green arrow represents the administration of hydroxocobalamin (vitamin B12). Red arrows represent time points at which tocilizumab was administered. As outlined, blood pressures were positively responsive to tocilizumab administration and pressors began to be weaned off after the first dose, quickly surpassing goal MAP $>65$ and maintaining stability until pressors could be discontinued

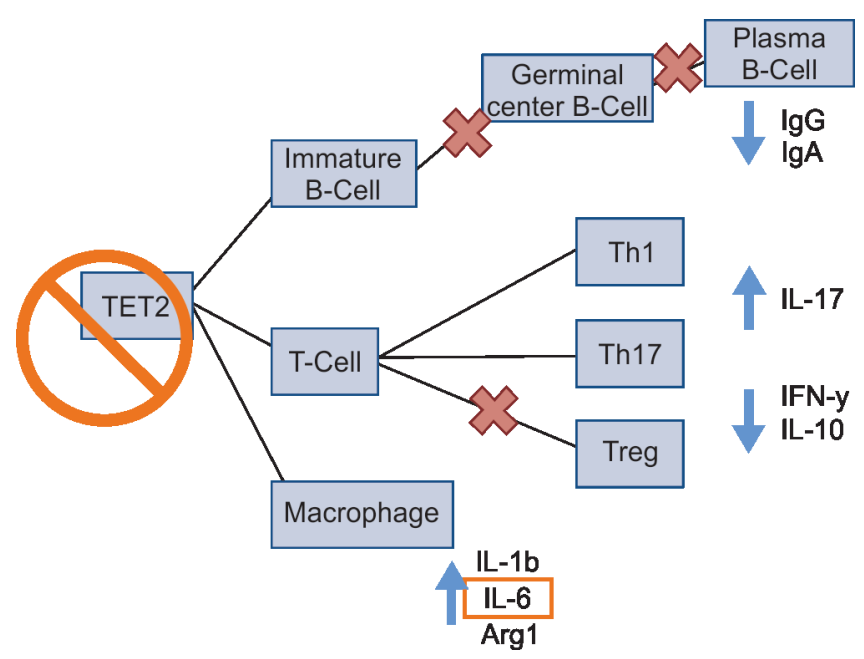

Fig. 2: Proposed mechanism of CMML TET2 mutation leading to increased expression of inflammatory cytokines. The TET2 mutation results in decreased germinal center B-cell, plasma B-cell, and regulatory $T$-cell proliferation, as indicated by the red " $X$ ". This consequently leads to a decrease in IgG, IgA, and IFN-gamma, while inactivating the TET2-mediated active repression of IL-6 transcription causing increased inflammatory cascades

Much of the literature documenting severe cases are associated with Bispecific T-cell engagers or chimeric antigen receptor T-cell immunologic therapies. Given known IL-6 elevations in CRS with relatively minimal significance in T-cell function otherwise, multiple trials demonstrated the efficacy of tocilizumab as treatment, gaining FDA approval in August 2017 in 
patients aged 2 years and older. ${ }^{4}$ Current recommendations also suggest the use of corticosteroids for counteracting the adverse neurologic effects of CRS within the context of such therapies given their superior capacity to traverse the blood-brain barrier. ${ }^{4}$

Furthermore, cardiovascular surgery has a known association with the development of SIRS, causing the release of IL-1, IL-6, IL-8, and TNF- $a$. $^{3,5,6}$ The magnitude of such cytokine release is also in direct correlation to the duration of ischemia in bypass surgery. ${ }^{1}$ Surgical trauma also stimulates local inflammatory cascades which cyclically engage clonally derived monocyte and granulocyte release from the bone marrow into the bloodstream circulation. This recruitment can propagate to a significant degree instigating a calamitous hyperinflammatory status characterized by leukostasis. ${ }^{6}$ Notably, the risk of leukostasis in CMML poorly correlates with the number of blasts in peripheral blood. ${ }^{7}$ Postperfusion syndrome has been described for decades, typically in association with cognitive injury or lung injury such as acute respiratory distress syndrome. Prior studies investigating CRS in patient populations undergoing CPB similarly demonstrated that IL- 6 was a key component in syndromic development and was the only cytokine correlating to pulmonary dysfunction. ${ }^{6}$ This elevation in IL-6 remained at 24 hours following bypass. ${ }^{3}$

\section{Conclusion and Highlights}

Existing literature data supporting IL-6 inhibitors as part of treating CRS were consequently extrapolated to a CMML patient developing SIRS involving CRS-like symptoms following CPB with much success. The additive state of these conditions led to a state similar in presentation to CRS. We thus described the novel combination of a CRS-like reaction in a CMML patient following CPB surgery treated successfully for the first time using tocilizumab.

\section{OrCID}

Wiaam Y Elkhatib 나 https://orcid.org/0000-0002-4706-1738

Hollie Saunders (0) https://orcid.org/0000-0003-1039-1108

Scott A Helgeson (ㄷ) https://orcid.org/0000-0001-7590-2293

John E Moss ㄴ https://orcid.org/0000-0003-1960-4819

\section{References}

1. Patnaik MM, Tefferi A. Chronic myelomonocytic leukemia: 2018 update on diagnosis, risk stratification and management. Am J Hematol 2018;93(6):824-840. DOI: 10.1002/ajh.25104.

2. Feng $Y$, Li X, Cassady K, Zou Z, Zhang X. TET2 function in hematopoietic malignancies, immune regulation, and DNA repair. Front Oncol 2019;9:210. DOI: 10.3389/fonc.2019.00210.

3. Passaroni AC, Felicio ML, Campos NLKL, Silva MAM, Yoshida WB. Hemolysis and Inflammatory response to extracorporeal circulation during on-pump CABG: comparison between roller and centrifugal pump systems. Braz J Cardiovasc Surg 2018;33(1):64-71. DOI: 10.21470/1678-9741-2017-0125.

4. Shimabukuro-Vornhagen $A$, Gödel $P$, Subklewe $M$, Stemmler $H J$, Schlößer HA, Schlaak M. Cytokine release syndrome. J ImmunoTher Cancer 2018;6(1):56. DOI: 10.1186/s40425-018-0343-9.

5. MoatNE, Shore DF, Evans TW. Organ dysfunction and cardiopulmonary bypass: the role of complement and complement regulatory proteins. Eur J Cardiothorac Surg 1993;7(11):563-573. DOI: 10.1016/10107940(93)90241-3.

6. Halter J, Steinberg J, Fink G, Lutz C, Picone A, Maybury R, et al. Evidence of systemic cytokine release in patients undergoing cardiopulmonary bypass. JECT 2005;37(3):272-277. PMID: 16350379; PMCID: PMC4680784.

7. Patel AB, Pettijohn EM, Abedin SM, Raps E, Deininger MW. Leukemoid reaction in chronic myelomonocytic leukemia patients undergoing surgery: perioperative management recommendations. Blood Adv 2019;3(7):952-955. DOI: 10.1182/ bloodadvances. 2019032300. 